\title{
Outcomes in children treated for persistent bacterial bronchitis
}

\author{
Deirdre Donnelly, Anita Critchlow, Mark L Everard
}

Thorax 2007;62:80-84. doi: 10.1136/thx.2006.058933

See end of article for authors' affiliations

....................

Correspondence to:

Dr M L Everard, Paediatric Respiratory Unit, Sheffield

Children's Hospital, Western Bank, Sheffield S1O 2TH,

UK;

m.l.everard@sheffield.ac.uk

Received 12 January 2006

Accepted 14 October 2006

Published Online First

13 November 2006
Background: Persistent bacterial bronchitis (PBB) seems to be under-recognised and often misdiagnosed as asthma. In the absence of published data relating to the management and outcomes in this patient group, a review of the outcomes of patients with PBB attending a paediatric respiratory clinic was undertaken.

Methods: A retrospective chart review was undertaken of 81 patients in whom a diagnosis of PBB had been made. Diagnosis was based on the standard criterion of a persistent, wet cough for $>1$ month that resolves with appropriate antibiotic treatment.

Results: The most common reason for referral was a persistent cough or difficult asthma. In most of the patients, symptoms started before the age of 2 years, and had been present for $>1$ year in $59 \%$ of patients. At referral, $59 \%$ of patients were receiving asthma treatment and $11 \%$ antibiotics. Haemophilus influenzae and Streptococcus pneumoniae were the most commonly isolated organisms. Over half of the patients were completely symptom free after two courses of antibiotics. Only $13 \%$ of patients required $\geqslant 6$ courses of antibiotics. Conclusion: PBB is often misdiagnosed as asthma, although the two conditions may coexist. In addition to eliminating a persistent cough, treatment may also prevent progression to bronchiectasis. Further research relating to both diagnosis and treatment is urgently required.
C oughing in childhood is common, with around a third of children reporting cough during any particular month, and of these a significant proportion will report the presence of a chronic cough. ${ }^{12}$ Despite being such a common symptom, there is remarkably little in the literature regarding the aetiology, investigation and management of chronic cough in childhood. Recent reports from Brisbane have emphasised the importance of making a specific diagnosis in children with a chronic cough (>3 weeks). ${ }^{3}$ In particular, they have highlighted the important prognostic implications of an ongoing wet cough and the importance of persistent bacterial bronchitis (PBB) as the most common cause of a chronic cough. ${ }^{3}{ }^{4} \mathrm{~A}$ variety of diagnostic labels have been used to describe this condition. Terms such as chronic suppurative lung disease, ${ }^{5-7}$ persistent endobronchial infection ${ }^{8}$ and $\mathrm{PBB}^{4}$ describe the pathological process and site of infection. Some use terms such as "chronic bronchitis"9-11 or "protracted bronchitis"12 13 to describe the clinical phenotype. Others have suggested using the term "pre-bronchiectasis"14 15 to highlight the condition's probable role in leading to damaged airways, as evident on high-resolution computed tomography (HRCT) or at bronchography. All these terms describe a condition that has received little attention during the past two decades. Indeed this important cause of chronic morbidity in otherwise healthy children is not mentioned in British paediatric respiratory text books, ${ }^{16}{ }^{17}$ although its clinical features are clearly described in an authoritative Australian text book. ${ }^{5}$ This is probably due to the intense focus on asthma that has dominated recent decades, and the lack of clarity in defining chronic bronchitis. The lack of agreement regarding terminology has been one of the major factors that has prevented further understanding of the natural history of this condition and has contributed to the lack of research into optimal approaches to treatment.

Consistent with the recently published data from Brisbane, ${ }^{34}$ $\mathrm{PBB}$ is the most common diagnosis made in our clinic among those referred with difficult asthma or with a chronic cough, and our impression is that the numbers presenting with PBB are increasing each year. This may be an unforeseen consequence of the substantial reduction in prescribing of antibiotics during the past decade. ${ }^{18-20}$
As the condition has received so little attention, it is not surprising that there is sparce information in the literature regarding treatment and outcomes. A review of the literature failed to identify any studies that would guide a clinician when treating this condition. Phelan et al suggest that antibiotic treatment "should be continued until the chest is clear and there are no symptoms". Our clinical experience and that of others, including the group from Brisbane, ${ }^{4}$ suggest that appropriate and aggressive medical treatment leads to a dramatic reduction in morbidity and complete resolution of symptoms in the vast majority of patients. This report contains the results of a retrospective review of outcomes in 81 randomly selected patients diagnosed with PBB.

\section{METHODS}

The notes of 81 patients, in whom a firm diagnosis of PBB had been made, were reviewed by a single member of the respiratory team (DD). The diagnosis was established using the standard criterion of a persistent, wet cough present for $>1$ month that resolves with appropriate antibiotic treatment. ${ }^{451213}$ This approach is directly analogous to the method of diagnosis of asthma, in which a diagnosis is made on the basis of history and clear response to appropriate treatment which can include reversibility with $\beta$-agonists or unequivocal dramatic improvement in symptoms when inhaled corticosteroids are started. Patients were randomly identified by reviewing clinic letters from the past 5 years. A database containing all clinic letters from this period was searched for the term "persistent endobronchial infection" (the term used in our clinic). This identified all patients in whom the diagnosis was considered to be part of the differential diagnosis. Patients' notes were then examined to determine whether the diagnosis had been confirmed and, if so, they were included in the notes review until the selected target of 80 sets of notes were examined. The patients were identified by a new member of the respiratory team (DD) to avoid recall bias that might be

Abbreviations: $H R C T$, high-resolution computed tomography; LRTI, lower respiratory tract infection; PBB, persistent bacterial bronchitis; PCD, polycystic disease 
introduced if patients had been identified from memory by doctors and nurses, which would probably have led to those with the most persistent symptoms being over-represented. Patients with significant neurological conditions such as severe cerebral palsy in whom such problems are relatively common and those who had undergone chemotherapy were excluded.

The approach to treatment was based on high-dose antibiotic treatment capable of treating Haemophilus influenzae and Streptococcus pneumoniae (most commonly amoxicillin with clavulanic acid $40 \mathrm{mg}$ amoxicillin content/day) for 2 weeks and reviewing response to treatment. If the cough cleared, the antibiotic was continued for a further 4-6 weeks in the first instance. Patients were discharged from the clinic when they had been through one autumn or winter without reoccurrence of the chronic cough.

A proforma was used to record all required information such as age of onset, symptoms at presentation, previous treatment(s), investigation results, response to prescribed treatment and current respiratory status. All data were then entered into an SPSS spreadsheet. Descriptive statistics were then analysed.

\section{RESULTS}

Most patients were referred from primary care, with a significant minority being referred from secondary care. The cohort included 41 girls and 40 boys aged 5 months to 14 years 9 months (median 3 years 9 months) when first seen in the clinic. Symptoms started before the age of 2 years in $59 \%$ of children (fig 1). The most common initiating event was an acute lower respiratory tract infection (LRTI; 42\%), with pneumonia accounting for a further $30 \%$. In the majority, this was a clinical diagnosis without documented $x$ ray changes. The most common reason for referral was a persistent cough or difficult asthma, accounting for approximately $47 \%$ and $45 \%$ of the group, respectively. At presentation $95 \%$ of patients had a persistent cough, usually described as "wet" or a "smoker's cough". "Wheeze" (48\%) and shortness of breath (43\%) were also common. Wheeze was the primary symptom in $5 \%$ of children, although on closer questioning almost all these children had a ruttle ${ }^{21}$ (a form of rale observed in young children, characterised by coarse discontinuous noises probably originating in the large airways) rather than classical wheeze. In many of those reporting shortness of breath, it was apparent that this was attributable to bouts of coughing. At least one parent smoked in $31 \%$ of families for whom a smoking history was available.

Symptoms had been present for $>1$ year in $59 \%$ of patients (fig 2). Most patients were receiving treatment at referral,

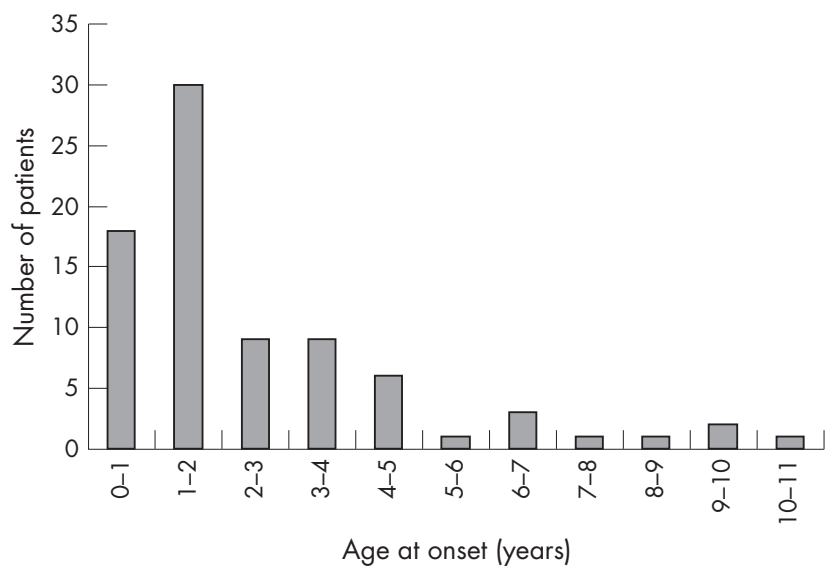

Figure 1 Age of onset of symptoms. including $48(59 \%)$ on asthma treatment (of these, 39 were taking inhaled corticosteroids) and $11 \%$ on antibiotics. A further $7.5 \%$ had been taking inhaled corticosteroids in the past. Only a small minority had abnormalities on auscultation, with coarse inspiratory and expiratory noises being the only abnormal findings. However, when requested to cough, $>95 \%$ had a wet cough.

Chest $x$ rays were carried out in $98 \%$ of the patients. Of these, $30 \%$ were reported as normal. The most common abnormality reported was bronchial wall thickening. Patchy consolidation was reported on at least one chest $x$ ray in $25 \%$ of the children. A further $19 \%$ had recurrent consolidation, with the same area being affected in nine children and different areas being affected in six. Bronchiectasis was confirmed in 4 of the 14 patients who had a HRCT scan. Of these, three had HRCT scans before, or immediately after, referral from local district general hospitals, the fourth having been referred with a foreign body that had been inhaled at least 6 months before referral. Two have since had repeat scans, having been asymptomatic for some time, and both show resolution of the original changes.

A cough swab was obtained in $63 \%$ of patients, and pathogens were grown in over half of the swabs. Of the infected samples, $H$ influenzae were cultured in $81 \%$ and $S$ pneumoniae in 37\%, (both were cultured in 30\%). Other organisms detected occasionally were moxarella and other streptococci.

Over half $(51 \%)$ of patients were completely symptom free after two courses of antibiotics. Only 13\% required six or more courses of antibiotics, or had continuous prophylactic antibiotics for at least one winter. At the time of the notes review all patients were well, although 5\% (4 patients) were still under active follow-up and receiving intermittent antibiotics. A firm diagnosis of concomitant asthma based on a bronchodilator response of $>15 \%$ forced expiratory volume in $1 \mathrm{~s}$ or a clear response to oral steroids had been made in $31 \%$ of patients.

Investigations were generally carried out only in those requiring more than two courses of antibiotics, and these were generally unhelpful. Immunoglobulin (Ig)G subclass abnormalities were detected in 28 of 36 patients, with raised and low levels being equally common. Only three patients had a low IgA level. Subclasses were normal in these patients and all three are currently symptom free. IgE was found to be raised in 10 of 35 patients. Antibody levels for Hib, tetanus and pneumococcus were checked in a third of the children. Only $41 \%$ of Hib levels were within the normal range, but almost all levels returned to normal after revaccination. With tetanus and pneumococcus, $69 \%$ and $70 \%$ had protective or normal levels, respectively. Other investigations, such as sweat tests and ciliary biopsies, were all negative.

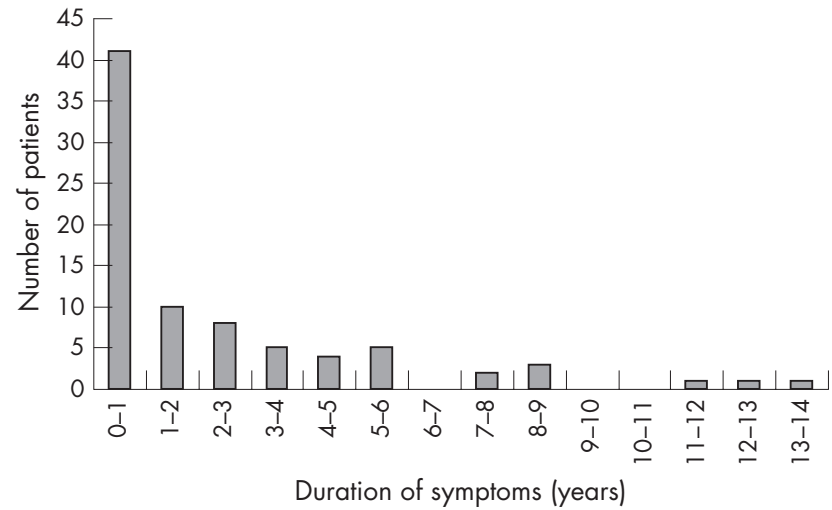

Figure 2 Duration of symptoms at diagnosis. 
Bronchoscopy was undertaken in 19 patients. Culture results were similar to cough swab results with $H$ influenzae followed by $S$ pneumoniae being the most commonly identified organisms. The cytology in lavage samples was dominated by neutrophils, which accounted for most cells. Other than secretions and oedematous mucosa, the only abnormality seen was malacic airways in two patients. Oedematous mucosa and closure of airways on suction during bronchoalveolar lavage was very common, but seemed to be secondary to the endobronchial inflammation.

\section{DISCUSSION}

The data presented in this report help to highlight the importance of making an accurate diagnosis in children with persistent cough and, in particular, a persistent wet cough. $95 \%$ of the children included in this study who presented with PBB were effectively cured with appropriate antibiotic treatment at the time of the notes review. However, a small number had a more protracted course even though they did not have an identifiable underlying cause such as cystic fibrosis, polycystic disese (PCD) or marked immunodeficiency.

Although this is a retrospective, observational study involving children with an established diagnosis of PBB, the data obtained from this study are similar to those in the prospective study from Marchant et $\mathrm{al}^{3}{ }^{4}$ in which 102 children with chronic persistent cough were put through a formal protocol. By far the most common diagnosis in their study was PBB, which accounted for $40 \%$ of patients, of whom $62 \%$ had an onset of symptoms in the first year of life. $54 \%$ had been labelled as having asthma, the others having been referred with persistent cough or bronchitis. The second most common diagnosis was bronchiectasis, which is likely to be a part of the PBB spectrum. As in our series, no significant underlying predisposing factor such as cystic fibrosis or PCD was identified, whereas bronchiectasis on HRCT was uncommon. The range of organisms identified was similar, with $H$ influenzae and $S$ pneumoniae accounting for $47 \%$ and $35 \%$ of isolates, respectively, and more than one organism being isolated from many patients. Most patients in this study became symptom free, although the report includes few details regarding treatment, other than noting that repeated courses of treatment were required in a significant number. Their patients seemed to have been symptomatic for a shorter period than ours, with a median duration of 6 months (interquartile range (IQR) 3-12).

Two other recent studies involving preschool children have further highlighted the prevalence of $\mathrm{PBB}$ in patients with troublesome respiratory symptoms. One of the studies identified bacterial bronchitis in $43 \%$ of children with persistent wheeze, despite adequate treatment for asthma (24\% were receiving oral steroids). ${ }^{22}$ In the same study, a significant number of children had coexisting reflux and positive bacterial cultures. Aspiration is likely to be an important predisposing condition in early childhood leading to disruption of the normal epithelium and colonisation by organisms such as $H$ influenzae. In a second study, a positive bacterial culture was obtained at bronchoscopy in $14(61 \%)$ of 23 children with recurrent cough and/or wheeze who had failed to respond to empirical treatment for asthma and gastro-oesophageal reflux. ${ }^{23}$

PBB has received little attention in recent decades, which is unfortunate as our experience, and that of the Brisbane group, is that, if recognised, it is a curable condition. Many centres are reluctant to accept this diagnosis as an important cause of cough in children. ${ }^{24}$ This may, in part, be attributable to confusion surrounding nomenclature, with many terms being used for the same condition. The benefits for the child by making an accurate diagnosis are likely to go well beyond eliminating the cough. In a significant proportion of patients, the lower airway inflammation accompanying PBB would probably progress inexorably over varying periods of time to "idiopathic bronchiectasis", as suggested by many authors over the years. ${ }^{5} 14{ }^{15}$ Clearly, it is difficult to prove this suggestion as it would be unethical to randomise children to effective treatment and placebo to observe the natural history. The suggestion that bronchiectasis represents a late stage in the progression of chronic bronchitis or PBB was first made in the 1940 s as a result of undertaking bronchography in children with chronic productive cough suggestive of bronchiectasis. ${ }^{14}$ Of those with a prolonged wet cough but without evidence of bronchiectasis at presentation, 40\% had developed clear evidence of bronchiectasis on repeat bronchography within 3 years. The author suggested that these children with chronic bronchitis had prebronchiectasis. Consistent with this suggestion is the observation that the clinical features of patients included in this report, and that of the Brisbane group, are remarkably similar to those outlined in recent studies involving children and adults with idiopathic bronchiectasis. It is reported that most adult patients give a history of an ongoing wet cough from early childhood. ${ }^{25}{ }^{26}$ It is also clear that many children $^{15} 27$ and adults ${ }^{25} 26$ are misdiagnosed as having conditions such as difficult asthma for some time before a diagnosis of bronchiectasis is made. Moreover, the widely accepted "vicious circle"28 29 hypothesis would predict that the development of bronchiectasis, as evident on HRCT, is preceded by a period of chronic inflammation driven by persistent bacterial infection of the conduction airways. Unfortunately, most publications relating to idiopathic bronchiectasis focus on the diagnosis and management of established bronchiectasis and ignore the antecedent stages of the disease. Focusing on bronchiectasis in this way is analogous to discussing the treatment of myocardial infarction and ignoring antecedent symptoms such as angina.

The levels of bronchiectasis in our clinic and this series are comparable with those of Marchant et al, ${ }^{4}$ but are much lower than those reported from other tertiary clinics. ${ }^{15} 270$ This may reflect our delay in proceeding to HRCT, and the relatively short duration of symptoms in Marchant et al's study. It has been known for decades that the appearance of bronchiectasis, on bronchography $^{31}$ or HRCT, ${ }^{5}$ can resolve with appropriate treatment, and this has been highlighted again in a recent study. ${ }^{15}$ This is entirely consistent with the proposition that the spectrum of PBB ranges from early colonisation, with minimal disruption of the airways, through to established bronchiectasis, which is simply a stage in the progression that can be identified radiologically on HRCT. PBB, without a significant underlying problem, seems curable, at least in the paediatric population. As in our experience the HRCT appearances do not affect the management or outcomes, we generally reserve HRCT for those with the most troublesome symptoms. In patients with PBB and a significant underlying condition such as cystic fibrosis, PCD or immunodeficiency, the progression to HRCT evident bronchiectasis is likely to be more rapid, and account for the high levels of identifiable predisposing factors present in those investigated for established bronchiectasis in childhood. ${ }^{27}$ Even in those with a significant immunodeficiency, resolution of bronchiectatic changes on HRCT is not uncommon with aggressive traetment (Wallis $\mathrm{C}$ personal communication).

The "vicious circle" hypothesis proposes that the initial event involves impairment of the normal pulmonary defense mechanisms, which allows inhaled bacteria such as $H$ influenzae to reside in the airways for longer than normal. ${ }^{28}{ }^{29}$ The most common insult in early childhood is likely to be viral LRTIs, which are known to disrupt normal surface morphology and cilial function for many weeks. ${ }^{32}$ The high incidence of PBB in the first few years of life, at a time when the prevalence of viral 
LRTIs is at their highest, would support this suggestion. In most studies attempting to identify a cause for bronchiectasis, "post LRTI" remains the commonest aetiology, with "unknown" being next. ${ }^{33}$ Previous "hospitalised pneumonia" is a major risk factor for bronchiectasis. ${ }^{15}{ }^{33}$ Our experience suggests that many with a chronic cough, which has been attributed to a "pneumonic illness", had the symptoms for some time before the pneumonic illness, which probably represented an acute exacerbation of PBB.

The significant number of patients with both asthma and PBB in our series is of interest. This has been observed in other reports in which the incidence of asthma in patients with bronchiectasis is significantly higher than the general population. ${ }^{34-37}$ It has been suggested that this is due to the inflammation associated with bronchiectasis causing reactive airways. However, it seems more likely that increased mucus secretion and mucus plugging, with disruption of the epithelium, leads to a predisposition to $\mathrm{PBB}$ consistent with the vicious circle hypothesis. The classical example of asthma and PBB coexisting is the so-called "right middle lobe syndrome", ${ }^{38}$ but, as with bronchiectasis, this probably represents the most easily defined example of a spectrum of severity. We are conscious that highlighting this condition carries the risk of overprescribing of antibiotics for patients with asthma. It is important to recognise that PBB is characterised by a prolonged moist cough and that antibiotics are not indicated for acute exacerbations of asthma.

As this entity has not been well described, there is no consensus regarding treatment. In the very early stages after the initial insult, standard, short courses of antibiotics may probably be sufficient to clear the organisms from the airway and allow healing. However, by the time patients have been referred to secondary care, symptoms have often been present for many months or years. Our approach is to attempt to eradicate infection and then to keep the airways clear of infection while the repair process takes place. Initially, we use a 2 -week course of an antibiotic such as amoxicillin with clavulanic acid at high dose and review the response at 2 weeks, an approach also adopted by Marchant et al. ${ }^{4}$ Typically, the cough takes 10-14 days to resolve. If there has been a clear response to this intervention, the treatment is continued for a further 4-6 weeks in order to keep the airways free from colonisation and allow repair of the affected airways. As noted in the Results section, most children remain symptom free after one or two such courses. The minority of patients seem to require prolonged courses, particularly through the winter, but even in these we generally observe resolution of symptoms after variable periods of time. For patients whose symptoms recur after two courses of antibiotics, physiotherapy is included in the treatment regimen. Other centres have reported using 3 or 6 months of antibiotics as their initial approach. Although none of the patients included in this report required intravenous treatment, we have found increasing need for this approach in the past 2 years. More recently we have been using nebulised antibiotics (colistin) for those with more troublesome symptoms. Indeed, colistin has been used very successfully in a small number whose cough has failed to resolve even with intravenous antibiotics. The ease with which symptoms are treated is likely to be influenced in part by the duration of symptoms. An adult study of PBB proved by bronchoscopy, without bronchiectasis on HRCT, found that even 3 weeks of oral antibiotics failed to clear the cough and all their patients required intravenous antibiotics. ${ }^{39}$

The progressive fall in prescribing antibiotics for viral respiratory tract infections in early childhood over the past decade $^{18-20}$ has been appropriate. However, this may have the consequence of inadvertently increasing the incidence of PBB, and possibly idiopathic bronchiectasis, in the coming decades. ${ }^{37}$ Our clinical impression is that PBB has become more common and difficult to treat over the past decade. Although there has been much emphasis on the reduction in prescribing antibiotics for upper respiratory tract infections in children, far less attention has been paid to those who might benefit from delayed prescribing. ${ }^{40}$ It has variously been suggested that prescribing antibiotics should be considered in patients with a persistent, wet cough lasting $>2,{ }^{40}{ }^{41} 4^{12}$ or $6^{9}$ weeks. We believe that prescribing antibiotics should be seriously considered if a wet cough persists for $>3-4$ weeks, provided that the possibility of mild asthma is considered. A careful clinical assessment is required to ensure that those with probable bacterial colonisation are treated appropriately, while avoiding a drift back to the treatment of asthma exacerbations and simple viral respiratory tract infections with antibiotics. Early in the course of PBB short courses of antibiotics are probably adequate, but, once established for months or years, longer courses are required to permit recovery of the epithelial integrity.

In summary, the diagnosis of $\mathrm{PBB}$ can be made at bronchoscopy or can be based on clinical assessment and response to treatment. Patient numbers seem to be increasing, which may be attributable to the reduction in inappropriate prescribing of antibiotics in the community, which has not been accompanied by the appropriate use of delayed prescribing. ${ }^{40}$ Diagnosis and appropriate treatment can lead to complete resolution of symptoms with dramatic improvements in quality of life. Identifying the underlying causes other than previous LRTIs in non-specialist clinics is uncommon, but causes should be sought in those with recurrent symptoms despite appropriate treatment. PBB is often misdiagnosed as asthma, leading to inappropriate and excessive use of inhaled steroids. Not uncommonly, the two conditions coexist, causing significant challenges in diagnosis and management.

Bronchiectasis has been termed an orphan disease owing to the relative lack of research into the diagnosis and management of this condition. ${ }^{372}$ There has been even less research into the accurate identification and management of children with PBB and pre bronchiectasis. Challenges for the future include establishing a universally agreed nomenclature, further clarifying diagnostic techniques, devising management algorithms and developing randomised clinical trials aimed at both PBB of relatively recent onset and for those with symptoms that have been present for many months or years.

\section{Authors' affiliations \\ Deirdre Donnelly, Anita Critchlow, Mark L Everard, Paediatric Respiratory Unit, Sheffield Children's Hospital, Western Bank, Sheffield, UK \\ Funding: DD was supported by the Sir Halley Stewart Trust \\ Competing interests: None.}

Ethical approval: This paper describes the outcome of an audit undertaken within the respiratory team through which we wished to get a handle on the outcomes achieved by our service. In the process of the audit, it became evident that there has been nothing published on treatment and outcomes for persistent bacterial bronchitis for more than half a century, and therefore we have written up the audit for publication. Because of its origins, this was not initially submitted for ethical approval as our initial aim was simply to quantify the effectiveness of our approach and treatment outcomes.

\section{REFERENCES}

1 Leonardi GS, Houthuijs D, Nikiforov B, et al. Respiratory symptoms, bronchitis and asthma in children of Central and Eastern Europe. Eur Respir $J$ 2002;20:890-8.

2 Kogan MD, Pappas G, Yu SM, et al. Over-the-counter medication use among US preschool-age children. JAMA 1994;272:1025-30.

3 Marchant JM, Masters IB, Taylor SM, et al. Utility of signs and symptoms of chronic cough in predicting specific cause in children. Thorax 2006;61:694-98. 
4 Marchant JM, Masters IB, Taylor SM, et al. Evaluation and outcome of young children with chronic cough. Chest 2006;129:1132-41.

5 Phelan PD, Landau LI, Robertson CF. Suppurative lung disease. In: Respiratory illness in children, 4th edn. Oxford, UK: Blackwell Scientific, 1994:295-06.

6 Chang AB, Boyce NC, Masters IB, et al. Bronchoscopic findings in children with non-cystic fibrosis chronic suppurative lung disease. Thorax 2002;57:935-8.

7 Couriel J. Assessment of the child with recurrent chest infections. Br Med Bull 2002;61:115-32.

8 Spencer DA. From hemp seed and porcupine quill to HRCT: advances in the diagnosis and epidemiology of bronchiectasis. Arch Dis Child 2005;90:712-14.

9 Field CE. Bronchiectasis in childhood III. Prophylaxis, treatment and progress with a follow up study of 202 cases of established bronchiectasis. Pediatrics 1949:4:355-72.

10 Morgan WJ, Taussig LM. The chronic bronchitis complex in children. Pediatr Clin N Am 1984;31:851-63.

11 Seear M, Wensley D. Chronic cough and wheeze in children: do they all have asthma. Eur Rspir J 1997;10:342-5.

12 Chang $A B$. Defining the cough spectrum and reviewing the evidence for treating non-specific cough in children. Curr Pediatr Rev 2005;1:283-96.

13 Chang A, Landau LI, Van Asperen PP, et al. Thoracic Society of Australia and New Zealand Position Statement. Cough in children: definitions and clinical evaluation. Med J Aust 2006;184:398-403.

14 Field CE. Bronchiectasis in childhood II. Aetiology and pathogenesis, including as survey of 272 cases of doubtful irreversible bronchiectasis. Pediatrics 1949;4:231-48.

15 Eastham KM, Fall AJ, Mitchell, et al. The need to redefine non-cystic fibrosis bronchiectasis in childhood. Thorax 2004;59:324-7.

16 Dinwiddie R. The diagnosis and management of paediatric respiratory disease. Edinburgh: Churchill Livingstone, 1990.

17 Silverman $M, \mathrm{O}^{\prime}$ Callaghan $\mathrm{CL}$, eds. Practical paediatric respiratory medicine. London: Arnold, 2001.

18 Linehan MF, Hazell ML, Frank TL, et al. Prevalence of respiratory symptoms in under 5s: 1993 to 2001. Arch Dis Child 2005;90:516-19.

19 Sharland M, Kendall H, Yeates D, et al. Antibiotic prescribing in general practice and hospital admissions for peritonsillar abscess, mastoiditis, and rheumatic fever in children: time trend analysis. BMJ 2005;331:328-9.

20 Arnold SR, Bush AJ. Decline in inappropriate antibiotic use over a decade by pediatricians in a Tennessee community. Ambul Pediatr 2006;6:225-9.

21 Elphick HE, Ritson S, Rodgers $\mathrm{H}$, et al. When a "wheeze" is not a wheeze: acoustic analysis of breath sounds in infants. Eur Respir J 2000;16:593-7.

22 Saglani S, Nicholson AG, Scallan M, et al. Investigation of young children with severe recurrent wheeze: any clinical benefit? Eur Respir J 2006;27:29-35.
23 Saito J, Harris WT, Gelfond J, et al. Physiologic, bronchoscopic, and bronchoalveolar lavage fluid findings in young children with recurrent wheeze and cough. Pediatr Pulmonol 2006;41:709-19.

24 Rubin BK. Pediatricians are not just small internists. Chest 2006;129:1118-21.

25 Pasteur MC, Helliwell SM, Houghton SJ, et al. An investigation into causative factors in patients with bronchiectasis. Am J Respir Crit Care Med 2000;162:1277-84.

26 Kelly MG, Murphy S, Elborn JS. Bronchiectasis in secondary care: a comprehensive profile of a neglected disease. Eur J Intern Med 2003;14:488-492.

27 Li AM, Sonnappa S, Lex C, et al. Non-CF bronchiectasis: does knowing the aetiology lead to changes in management? Eur Respir J 2005;26:8-14.

28 Cole PJ, Wilson R. Host-microbial interrelationships in respiratory infection. Chest 1989:95:217S-21S.

29 Cole, P. The damaging role of bacteria in chronic lung infection. J Antimicrob Chemother 1997;40(Suppl A):5-10.

30 Twiss J, Metcalfe R, Byrnes CA. New Zealand national incidence of bronchiectasis "too high" for a developed country. Arch Dis Child 2005;90:736-40.

31 Field CE. Bronchiectasis in childhood I. Clinical survey of 160 cases. Pediatrics 1949:4:21-45

32 Wong JY, Rutman A, O'Callaghan C. Recovery of the ciliated epithelium following acute bronchiolitis in infancy. Thorax 2005;60:582-7.

33 Byrnes C. Non cystic fibrosis bronchiectasis. Paediatr Respir Rev 2006;7(Suppl 1):S255-7.

34 Saynajakangas $\mathbf{O}$, Keistinen $\mathrm{T}$, Tuuponen $\mathrm{T}$, et al. Links between hospital diagnoses of bronchiectasis and asthma. Allergy 1997;52:1 120-2.

35 Ip MS, So SY, Lam WK, et al. High prevalence of asthma in patients with bronchiectasis in Hong Kong. Eur Respir J 1992:5:418-23.

36 Singleton R, Morris A, Redding G, et al. Bronchiectasis in Alaska Native children: causes and clinical courses. Pediatr Pulmonol 2000;29:182-7.

37 Callahan CW, Redding GJ. Bronchiectasis in children: orphan disease or persistent problem? Pediatr Pulmonol 2002;33:492-6.

38 Springer C, Avital A, Noviski N, et al. Role of infection in the middle lobe syndrome in asthma. Arch Dis Child 1992;67:592-4.

39 Schaefer OP, Irwin RS. Unsuspected bacterial suppurative disease of the airways presenting as chronic cough. Am J Med 2003;114:602-6.

40 Little P. Delayed prescribing of antibiotics for upper respiratory tract infection. BMJ 2005;331:301-2.

41 Phelan PD, Landau LI, Robertson CF. Clinical patterns. In: Respiratory illness in children, 4th edn. Oxford, UK: Blackwell Scientific, 1994:68.

42 Keistinen T, Saynajakangas O, Tuuponen T, et al. Bronchiectasis: an orphan disease with a poorly-understood prognosis. Eur Respir J 1997;10:2784-7. 